\title{
A LOW RANK AND SPARSE PARADIGM FREE MAPPING ALGORITHM FOR DECONVOLUTION OF FMRI DATA
}

\author{
Eneko Uruñuela ${ }^{\star \dagger}$, Stefano Moia ${ }^{\star \dagger}$, César Caballero-Gaudes ${ }^{\star}$ \\ *Basque Center on Cognition, Brain and Language, San Sebastián-Donostia, Spain \\ $\dagger$ University of the Basque Country, San Sebastián-Donostia, Spain
}

\begin{abstract}
Current deconvolution algorithms for functional magnetic resonance imaging (fMRI) data are hindered by widespread signal changes arising from motion or physiological processes (e.g. deep breaths) that can be interpreted incorrectly as neuronal-related hemodynamic events. This work proposes a novel deconvolution approach that simultaneously estimates global signal fluctuations and neuronalrelated activity with no prior information about the timings of the blood oxygenation level-dependent (BOLD) events by means of a low rank plus sparse decomposition algorithm. The performance of the proposed method is evaluated on simulated and experimental fMRI data, and compared with state-of-the-art sparsity-based deconvolution approaches and with a conventional analysis that is aware of the temporal model of the neuronal-related activity. We demonstrate that the novel low-rank and sparse paradigm free mapping algorithm can estimate global signal fluctuations related to motion in our task, while estimating the neuronal-related activity with high fidelity.
\end{abstract}

Index Terms - functional MRI, deconvolution, low rank and sparse models, paradigm free mapping.

\section{INTRODUCTION}

Deconvolution algorithms of functional magnetic resonance imaging (fMRI) data aim to estimate blood oxygenation level-dependent (BOLD) events with no prior knowledge of their timing. These algorithms can be specially useful when the information about the timing of the neuronal activity that drives the BOLD events is unknown, inaccurate or insufficient (e.g. resting-state, naturalistic paradigms or clinical conditions). However, the performance of existing deconvolution approaches can be hampered considerably in presence of global, widespread signal changes due to head jerks, hardware artefacts or prominent non-neuronal physiological events (e.g. deep breaths) [1]. These global events are difficult to compensate during data preprocessing [2] and can be misinterpreted as neuronally related since their temporal signature can closely resemble the hemodynamic response function (HRF) assumed in the deconvolution model to describe neurovascular coupling.

Most of the deconvolution algorithms adopt a simple linear timeinvariant model to estimate the neuronal-related signal by solving a temporal regularized least-squares problem, either using $\ell_{2}$ - or $\ell_{1}$ norm terms, that operates at the voxel level [3-5], although a certain spatial smoothness can also be considered [6].

This work was supported by the European Union's Horizon 2020 research and innovation program (Marie Skłodowska-Curie grant agreement No. 713673), La Caixa Foundation (ID 100010434, fellowship code LCF/BQ/IN17/11620063), the Spanish Ministry of Economy and Competitiveness (RYC-2017-21845), the Basque Government (BERC 2018-2021, PIBA_2019_104, PRE_2019_1_0054), the Spanish Ministry of Science, Innovation and Universities (PID2019-105520GB-100).
The aim of this work is to propose a new approach method for the spatio-temporal deconvolution of fMRI data that is capable of simultaneously estimating global signal fluctuations and neuronalrelated activity based on the low-rank plus sparse matrix decomposition method [7]. We extend the formulation of our original sparse paradigm free mapping (SPFM) [5] approach by using a regularized estimator consisting of a structured sparsity promoting $\ell_{2,1}+\ell_{1}$ norm and a low-rank promoting nuclear-norm, which allows the simultaneous spatio-temporal mapping of global fluctuations and neuronal-related activity without prior information about the timings of these events, hence providing a cleaner estimation of the neuronal-related activity.

\section{LOW-RANK AND SPARSE PARADIGM FREE MAPPING}

Let us consider that the whole-brain fMRI data $\mathbf{Y} \in \mathbb{R}^{N \times V}$ where $N$ is the number of volumes and $V$ is the number of voxels of the acquisition can be decomposed into three terms, i.e.

$$
\mathbf{Y}=\mathbf{H S}+\mathbf{L}+\mathbf{N}
$$

where the neuronal-related component $\mathbf{H S}$ is the convolution of voxel-specific neuronal-related signals $\mathbf{S}$ with the Toeplitz matrix $\mathbf{H} \in \mathbb{R}^{N \times N}$ with shifted HRFs in its columns (i.e. similar to paradigm free mapping [5]), the global fluctuations can be captured as the sum of $P$ spatially widespread (i.e. global) low-rank components $\mathbf{L}=\sum_{p=1}^{P} \mathbf{v}_{p} \mathbf{a}_{p}^{T}$ where $\mathbf{v}_{p} \in \mathbb{R}^{N \times 1}$ and $\mathbf{a}_{p} \in \mathbb{R}^{V \times 1}$ denote their corresponding spatial and temporal signatures, and $\mathbf{N}$ represents additional white Gaussian noise.

To estimate both the neuronal-related signals and the global components, we propose to solve the following multivariate regularized least-squares problem:

$$
\begin{aligned}
\hat{\mathbf{L}}, \hat{\mathbf{S}}=\arg \min _{\mathbf{L}, \mathbf{S}} \| \mathbf{Y}- & \mathbf{H S}-\mathbf{L}\left\|_{F}^{2}+\lambda_{L}\right\| \mathbf{L} \|_{*} \\
& +(1-\rho)\left\|\mathbf{S} \mathbf{D}_{S}\right\|_{2,1}+\rho\left\|\mathbf{S D}_{S}\right\|_{1},
\end{aligned}
$$

where $\|\cdot\|_{F}$ denotes the Frobenious norm, the $\ell_{2,1}+\ell_{1}$-norm term enforces temporal sparsity and spatial structure on the estimate of the neuronal-related activity and $\rho$ controls the tradeoff between both terms [8], $\mathbf{D}_{s}=\operatorname{diag}\left(\lambda_{S_{1}}, \ldots, \lambda_{S_{V}}\right)$ is diagonal matrix with voxelspecific regularization parameters that balances the sparsity of $\mathbf{S}$ and data fidelity for each voxel, and the nuclear-norm $\|\cdot\|_{*}$ encourages the estimation of low-rank components where $\lambda_{L}$ controls the number of low-rank components.

Here, we empirically set $\rho=0.8$ to enforce structure in the spatial domain and maintain the sparsity of the estimates. For each voxel, $\lambda_{S_{i}}$ is set equal to the median absolute deviation estimate of the noise standard deviation from the fine-scale wavelet coefficients of the voxel time series (Daubechies, order 3). After the singular 


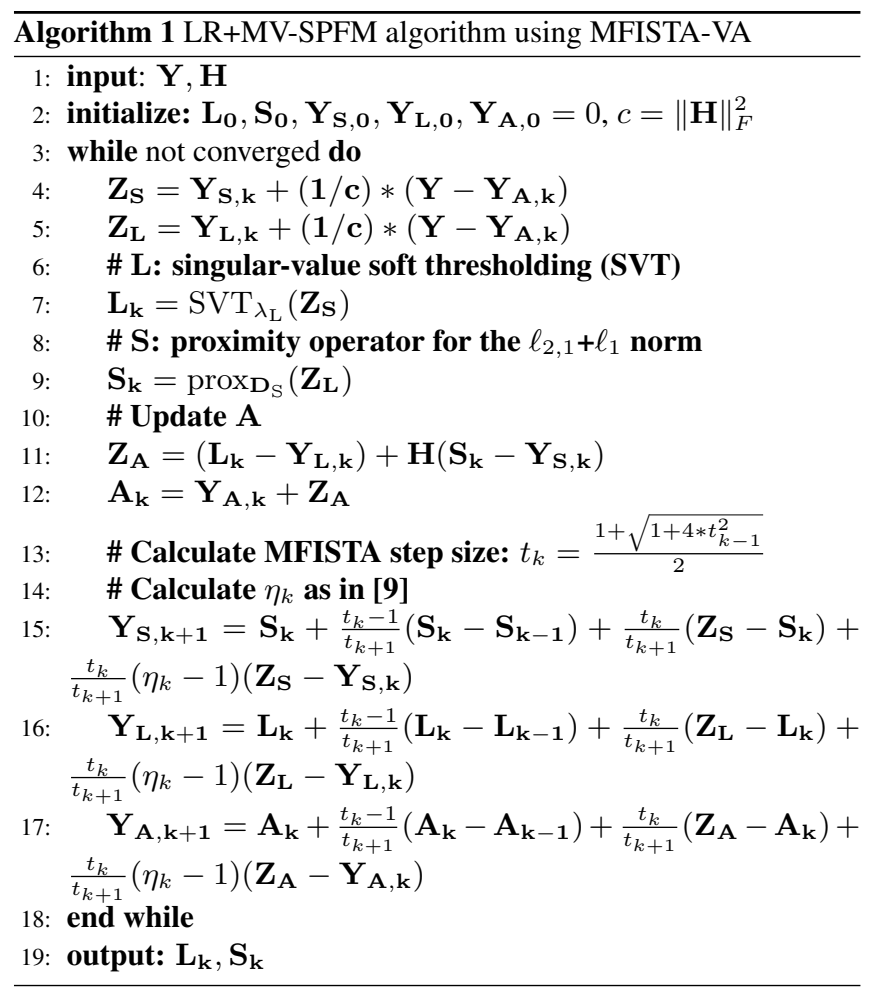

value decomposition (SVD) of the data, we set $\lambda_{L}$ to select $P$ lowrank components corresponding to the largest eigenvalues showing a difference of at least $10 \%$ with respect to the next eigenvalue. The optimization problem in 2 is solved via monotone FISTA with variable acceleration (MFISTA-VA) [9] as shown in Algorithm 1.

\section{RESULTS}

\subsection{Simulated data}

We simulated 1000 voxels including two groups of 50 voxels with a known BOLD signal, whereas the remaining voxels did not contain any BOLD signal. For each voxel, we added noise of different sources (motion-related, thermal and physiological noise) [5], as well as two global low-rank components (see Figure 1A) with a random voxelwise amplitude simulating widespread signal changes due to two deep breaths [2] and motion-related spikes, respectively. The performance of the proposed low-rank and multivariate sparse paradigm free mapping (LR+MV-SPFM) algorithm was asssesed on different signal to noise ratio (SNR) settings and with different ratios of BOLD/Total number of voxels, and benchmarked four different variations against the original univariate SPFM algorithm with regularization parameter selected according to the Bayesian Information Criterion (BIC) [5].

Figure 1B depicts the receiver operating characteristic (ROC) curves with the sensitivity and specificity for the estimation of the neuronal-related signal $\hat{\mathbf{S}}$ for each simulation scenario. Regardless of the simulated SNR and the BOLD/total voxels ratios, the ROC curves demonstrate the proposed LR+MV-SPFM algorithm is more specific and provides higher sensitivity in comparison with the original SPFM method, except with the highest BOLD/total number of voxels ratio and highest SNR where it seems that the multivariate nature of the model prevents the algorithm from fitting accurately at the voxel level. As expected, all variations of the proposed algorithm exhibit lower sensitivity as the SNR is reduced while maintaining the level of specificity. In addition, Figure $1 \mathrm{C}$ plots the error of the low-rank component estimate obtained with the LR+MV-SPFM algorithm for $\rho=0.8$, showing that its estimate improves with a lower BOLD-only / total voxels ratio.

\subsection{Experimental data}

Nine healthy subjects were scanned in a 3T MR scanner (Siemens) in ten MRI sessions. T2*-weighted multi-echo fMRI data was acquired with a multiband (MB) multiecho gradient echo planar imaging sequence (340 scans, 52 slices, Partial-Fourier=6/8, voxel size $=2.4 \times 2.4 \times 3 \mathrm{~mm}^{3}, \mathrm{TR}=1.5 \mathrm{~s}$, TEs $=10.6 / 28.69 / 46.78 / 64.87 / 82.96$ $\mathrm{ms}$, flip angle $=70^{\circ}, \mathrm{MB}$ factor $=4$, GRAPPA=2). During the fMRI acquisition, subjects performed a motor task consisting of five different movements (left-hand finger tapping, right-hand finger tapping, moving the left toes, moving the right toes and moving the tongue). These conditions were randomly intermixed every 16 seconds, and were only repeated once the entire set of stimuli were presented. For this work, only the first two sessions were selected to evaluate the algorithm. Data preprocessing consisted of optimally combining the echo time datasets, detrending of up to $5^{\text {th }}$-order Legendre polynomials, spatial smoothing ( $3 \mathrm{~mm}$ FWHM) and normalization to signal percentage change. The analysis was performed with the novel LR+MV-SPFM algorithm with $\rho=0.8$ and the selection of $\lambda_{L}$ and $\mathbf{D}_{S}$ described in section 2 .

Figures 2A-F depict the results of the LR+MV-SPFM algorithm in a representative dataset. For this subject and session, the proposed approach estimated $P=3$ global low-rank components whose time series $\mathbf{a}_{p}$ and spatial maps $\mathbf{v}_{p}$ are shown in Figures $2 \mathrm{C}$ and $2 \mathrm{~F}$, respectively. Figure 2A shows the Euclidean norm of the motion displacements (E-norm), DVARS and global signal (GS) time series, whereas 2B displays the grayplots of the preprocessed data (RAW), estimated low-rank component and estimated neuronal-related component in grey matter (GM) and white matter (WM) voxels. The first low-rank component captures signal fluctuations related to head movements and susceptibility artefacts during the 'moving the tongue' condition, suggesting that the subject moved the head while performing the tongue movement task. The second low-rank component has a time series that closely follows the global signal and its spatial map actually delineates major arteries and draining veins that strongly contribute to the global signal, whereas the third component is clearly related to global physiological fluctuations. Among participants, the number of low-rank components ranged between 1 and 5 .

Furthermore, Figures $2 \mathrm{D}$ and $2 \mathrm{E}$ illustrate the time series of the estimated neuronal-related signal for a representative voxel (see cross in the first map) and the maps for several individual events of the tongue movements and right hand finger tapping conditions, respectively. The LR+MV-SPFM maps reveal clusters of activity in similar regions to those inferred with general linear model analyses $(p<0.001)$. Notably, the LR+MV-SPFM maps still depict the tongue areas of the motor cortex bilaterally despite the timing of the first low-rank component followed the tongue condition.

Finally, Figure $2 \mathrm{G}$ depicts the ROC curves of the MV-SPFM and LR+MV-SPFM, both using $\rho=0.8$, and the original SPFM algorithm using the GLM maps of each event thresholded at a $p=0.001$ as the ground-truth. The ROC curves of the five motor task conditions show that both the MV-SPFM and the LR+MV-SPFM approaches provide higher sensitivity at the cost of a reduced specificity, and that the higher complexity involved in estimating the low- 
A



C

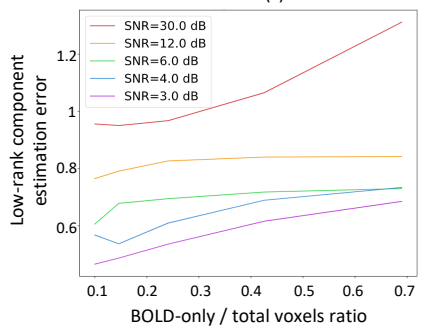

B

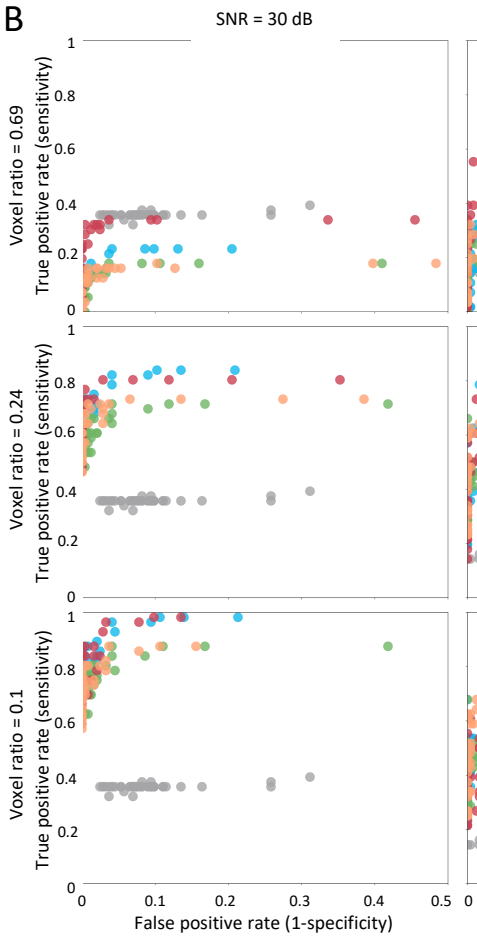

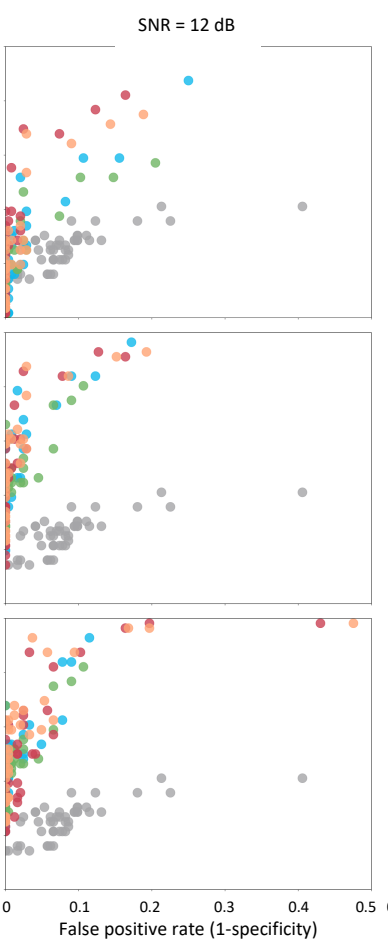

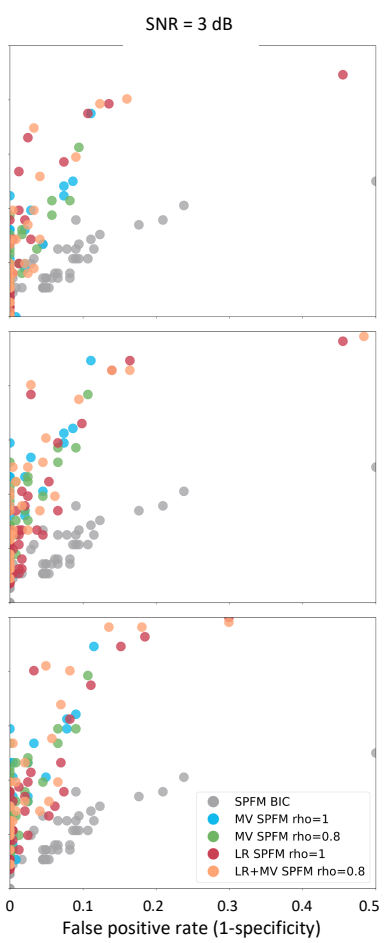

Fig. 1. Simulation results. A) An example of the simulated signals for the different SNR conditions; B) ROC curves for the estimation of the neuronal-related signal with: SPFM using BIC (SPFM BIC), SPFM with no low-rank estimation and no spatial regularization (SPFM, $\rho=1$ ), MV-SPFM with no low-rank estimation (MV-SPFM, $\rho=0.8$ ), the LR+MV-SPFM algorithm with only the L1-norm (LR+SPFM, $\rho=1)$, and the LR+MV-SPFM algorithm $(\rho=0.8)$. C) Estimation error of the low-rank components for different ratios of BOLD/total number of voxels.

rank component does not diminish the accuracy in deconvolving the neuronal-related component of the signal.

\section{CONCLUSIONS}

This work introduces a novel formulation for the deconvolution of BOLD fMRI data using a low rank and sparse algorithm that captures global fluctuations due to motion artefacts or physiological signals that typically reduce the accuracy of neuronal related estimates of currently used algorithms.

Future work will consider the evaluation on resting-state data, robust approaches for selecting the regularization parameters [10], extending the proposed formulation for multi-echo acquisitions [11] and describing the neuronal-related signal in terms of its innovation signals $[6,12]$.

\section{COMPLIANCE WITH ETHICAL STANDARS}

This study was approved by the institutional review board of the Basque Center on Cognition, Brain and Language.

\section{REFERENCES}

[1] Jonathan D. Power, Mark Plitt, Timothy O. Laumann, and A. Martin, "Sources and implications of whole-brain fMRI signals in humans," NeuroImage, vol. 146, pp. 609-625, 2017.
[2] Jonathan D. Power, M. Plitt, S. J. Gotts, P. Kundu, V. Voon, P. Bandettini, and A. Martin, "Ridding fMRI data of motionrelated influences: Removal of signals with distinct spatial and physical bases in multiecho data," Proceedings of the National Academy of Sciences, vol. 115, pp. E2105 - E2114, 2018.

[3] Darren R Gitelman, William D Penny, John Ashburner, and Karl J Friston, "Modeling regional and psychophysiologic interactions in fMRI: the importance of hemodynamic deconvolution," Neuroimage, vol. 19, no. 1, pp. 200-207, 2003.

[4] Ildar Khalidov, Jalal Fadili, François Lazeyras, Dimitri Van De Ville, and Michael Unser, "Activelets: Wavelets for sparse representation of hemodynamic responses," Signal processing, vol. 91, no. 12, pp. 2810-2821, 2011.

[5] César Caballero-Gaudes, Natalia Petridou, Susan T Francis, Ian L Dryden, and Penny A Gowland, "Paradigm free mapping with sparse regression automatically detects single-trial functional magnetic resonance imaging blood oxygenation level dependent responses," Human brain mapping, vol. 34, no. 3, pp. 501-518, 2013.

[6] Fikret Işık Karahanoğlu, César Caballero-Gaudes, François Lazeyras, and Dimitri Van De Ville, "Total activation: fMRI deconvolution through spatio-temporal regularization," $\mathrm{Neu}$ roimage, vol. 73, pp. 121-134, 2013. 
A
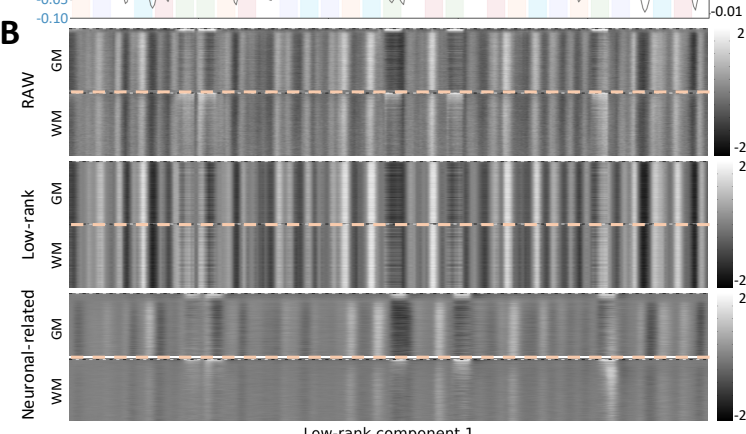

C

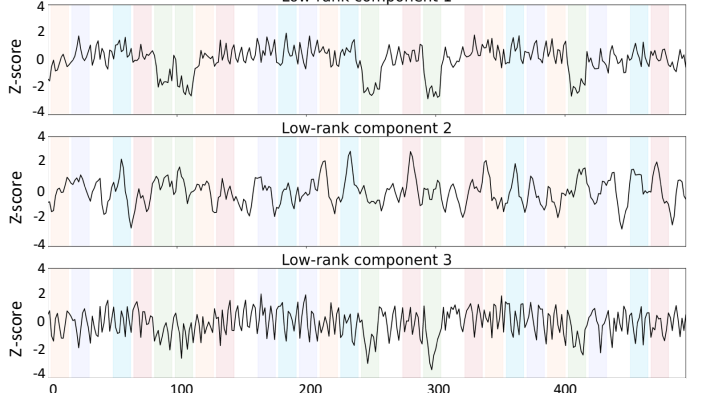

G

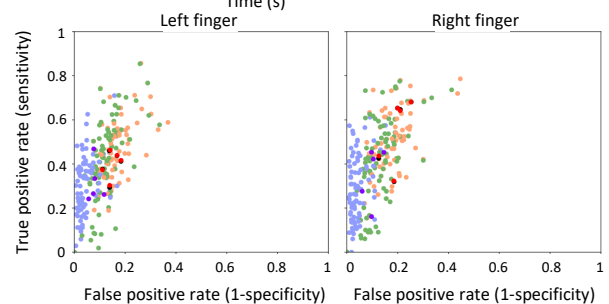

D

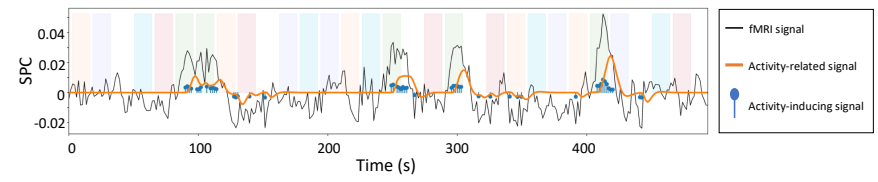

$\mathbf{E}$

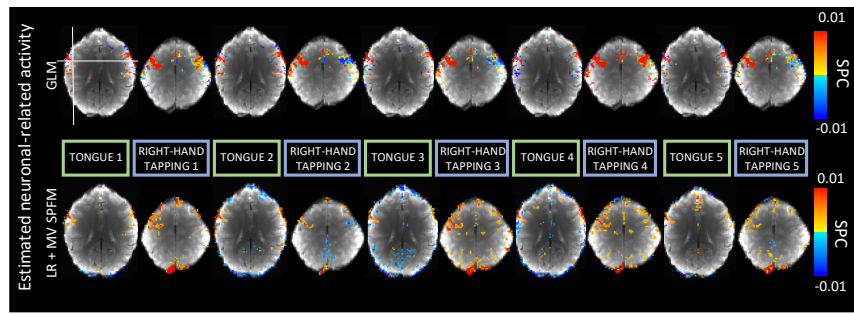

$\mathbf{F}$
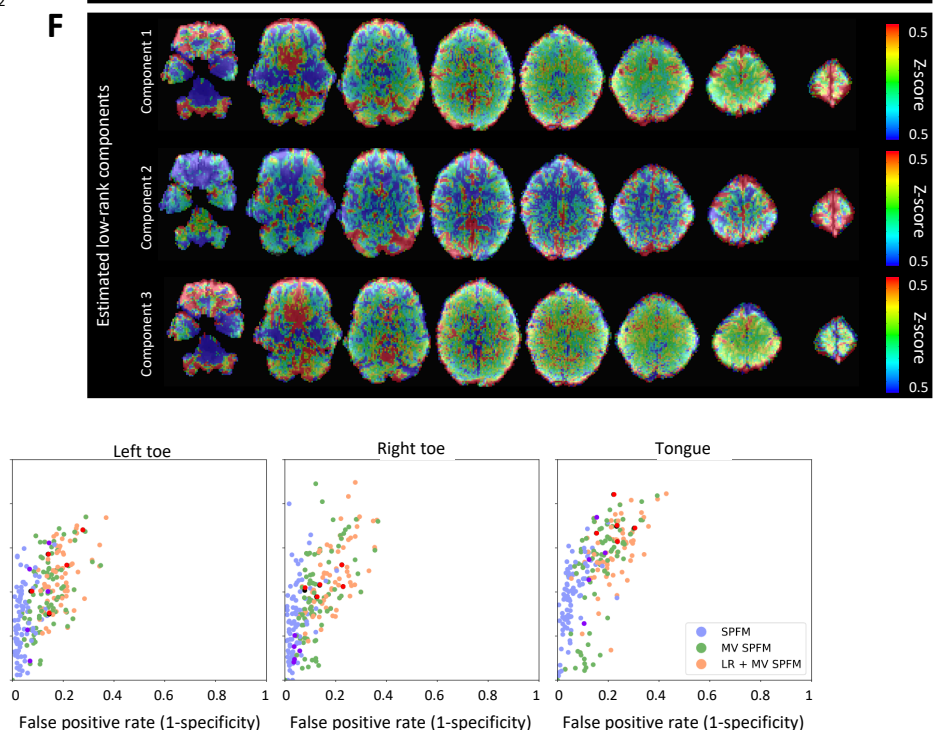

False positive rate (1-specificity) False positive rate (1-specificity) False positive rate (1-specificity)

Fig. 2. A) Euclidean norm of the motion displacements (E-norm) (blue), DVARS (black) and global (grey) signals of the fMRI data; B) Grayplots of grey matter (GM) and white matter (WM) of the preprocessed fMRI data, the estimated low-rank and neuronal-related components; C) Time series and F) maps of the estimated low-rank components; D) Time series and E) maps of the neuronal-related activity. G) ROC curves of the five conditions for the three algorithms tested: SPFM, MV SPFM and LR+MV SPFM (red, dark-purple and dark-green dots correspond to the subject in Fig. 2). The colour bands in the plots with time series denote the timing of the different conditions.

[7] Ricardo Otazo, Emmanuel Candes, and Daniel K Sodickson, "Low-rank plus sparse matrix decomposition for accelerated dynamic MRI with separation of background and dynamic components," Magnetic resonance in medicine, vol. 73, no. 3, pp. 1125-1136, 2015.

[8] Alexandre Gramfort, Daniel Strohmeier, Jens Haueisen, Matti Hamalainen, and Matthieu Kowalski, "Functional brain imaging with M/EEG using structured sparsity in time-frequency dictionaries," in Biennial International Conference on Information Processing in Medical Imaging. Springer, 2011, pp. 600-611.

[9] Marcelo Victor Wust Zibetti, Elias Salomao Helou, Ravinder R Regatte, and Gabor T Herman, "Monotone FISTA with variable acceleration for compressed sensing magnetic resonance imaging," IEEE transactions on computational imaging, vol. 5, no. 1, pp. 109-119, 2018.
[10] Eneko Uruñuela, Stephen Jones, Anna Crawford, Wanyong Shin, Sehong Oh, Mark Lowe, and César Caballero-Gaudes, "Stability-Based Sparse Paradigm Free Mapping Algorithm for Deconvolution of Functional MRI Data," in 2020 42nd Annual International Conference of the IEEE Engineering in Medicine \& Biology Society (EMBC). IEEE, 2020, pp. 1092-1095.

[11] César Caballero-Gaudes, Stefano Moia, Puja Panwar, Peter A Bandettini, and Javier Gonzalez-Castillo, "A deconvolution algorithm for multi-echo functional MRI: Multi-echo Sparse Paradigm Free Mapping," NeuroImage, vol. 202, pp. 116081, 2019.

[12] Hamza Cherkaoui, Thomas Moreau, Abderrahim Halimi, and Philippe Ciuciu, "Sparsity-based blind deconvolution of neural activation signal in fMRI," in ICASSP 2019-2019 IEEE International Conference on Acoustics, Speech and Signal Processing (ICASSP). IEEE, 2019, pp. 1323-1327. 